\title{
The disk/jet connection in the enigmatic microquasar Cygnus X-3
}

\author{
Karri I. I. Koljonen ${ }^{1}$, Diana C. Hannikainen ${ }^{1,2}$, Michael L. \\ McCollough $^{3}$, Guy G. Pooley ${ }^{4}$, Sergei A. Trushkin ${ }^{5}$, Marco Tavani ${ }^{6}$ \\ and Robert Droulans ${ }^{7}$ \\ ${ }^{1}$ Aalto University Metsähovi Radio Observatory, Metsähovintie 114, 02540 Kylmälä, Finland \\ email: karri@kurp.hut.fi \\ ${ }^{2}$ Finnish Centre for Astronomy with ESO (FINCA), University of Turku, Väisäläntie 20, \\ FI-21500 Piikkiö, Finland \\ ${ }^{3}$ Smithsonian Astrophysical Observatory, 60 Garden Street, Cambridge, MA 02138-1516, USA \\ ${ }^{4}$ Astrophysics Group, Cavendish Laboratory, 19 J. J. Thomson Avenue, Cambridge CB3 0HE, \\ UK \\ ${ }^{5}$ Special Astrophysical Observarory RAS, Karachaevo-Cherkassian res, Nizhnij Arkhyz, 36916, \\ Russia \\ ${ }^{6}$ INAF-IASF, I-00133 Rome, Italy \\ ${ }^{7}$ CESR/CNRS - Université de Toulouse, 9 Av. du Colonel Roche, 31028 Toulouse Cedex 04, \\ France
}

\begin{abstract}
Simultaneous multi-wavelength observations are crucial for understanding the physics of microquasars, especially the accretion disk/jet connection. The enigmatic microquasar Cygnus X-3 exhibits strong, relativistic jet ejection events producing radio flares up to $20 \mathrm{Jy}$. These events are preceded by a very soft X-ray state with quenched emission in the radio and hard X-ray bands. Recently, GeV flux was observed by the AGILE and Fermi $\gamma$-ray observatories during the newly-identified hypersoft state. By using an extensive database of simultaneous multi-wavelength observations gathered from Cygnus X-3 we can form a more unified picture of the nature of the source and show how the recent $\gamma$-ray observations fit into it.
\end{abstract}

Keywords. Accretion, accretion disks - Binaries: close - Gamma rays: observations - Radio continuum: stars - Stars: winds, outflows $-\mathrm{X}$-rays: binaries $-\mathrm{X}$-rays: individual: Cygnus X-3

\section{Introduction}

Cygnus X-3 (Cyg X-3) is a well-known X-ray binary (XRB) discovered in 1966 (Giacconi et al. 1967) but whose true nature remains a mystery despite extensive multiwavelength observations throughout the years. Its X-ray lightcurve (Parsignault et al. 1972) shows strong 4.8-hour orbital modulation and the infrared lightcurve (Mason et al. 1986) weaker modulation, typical of low-mass XRBs. However, infrared observations suggest that its mass-donating companion is a Wolf-Rayet (WR) star (van Kerkwijk et al. 1992), which would make it a high-mass XRB. Also, unlike most other XRBs, Cyg X-3 is relatively bright in the radio virtually all of the time and it undergoes giant radio outbursts with strong evidence of jet-like structures moving away at relativistic speeds (e.g. Mioduszewski et al. 2001). The nature of the compact object is not certain, but it is thought to be a black hole due to its spectral resemblance to other black hole XRB systems, such as GRS 1915+105 and XTE J1550-564. In addition, there is no evidence of a neutron star system producing such massive radio outbursts (up to $\sim 20 \mathrm{Jy}$, Waltman et al. 1995) as observed from Cyg X-3. Cyg X-3 is a unique system in our Galaxy, but 


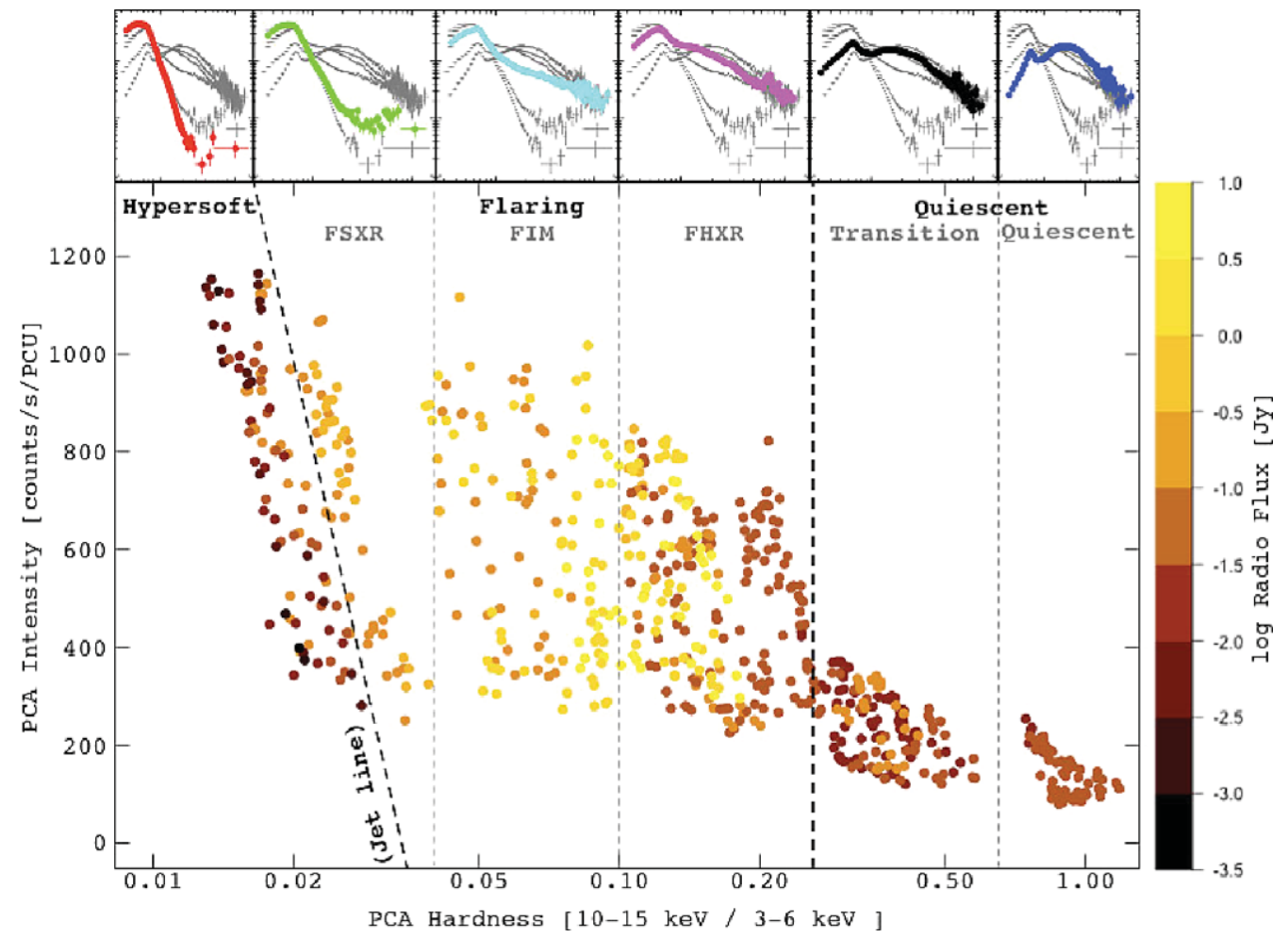

Figure 1. The hardness-intensity diagram showing the added information gained when including the radio dimension (Koljonen et al. 2010). The radio flux is represented by the hue of the points, with lighter colors referring to higher flux and darker to lower flux. The six different spectral states of Cyg X-3 are clearly demarcated in the plot, and the X-ray spectra are plotted above their respective areas. The jet line, between the hypersoft and the flaring soft X-ray state, is marked: a major flare occurs when the source transits across this line from the hypersoft state to the FSXR state.

recent observations of two XRBs in IC 10 (Prestwich et al. 2007) and NGC 300 (Carpano et al. 2007), both containing a WR companion, show strong evidence of a black hole primary. It is worth noting that these systems may represent a crucial link towards the evolution of a double black hole binary.

Here we shall review the radio/X-ray states of Cyg X-3 and show where the GeV emission fits into this picture.

\section{Unified picture of Cyg X-3: the radio/X-ray states}

It was clear early on that in order to make sense of the spectral evolution of Cyg X-3, multi-wavelength observations are necessary (e.g. McCollough et al. 1999; Szostek, Zdziarski \& McCollough 2008; Koljonen et al. 2010). In Fig. 1 (reproduced from Koljonen et al. 2010), the HID for Cyg X-3 is plotted using additional data from (near) simultaneous radio coverage. In this way the radio/X-ray states can be divided according to the $\mathrm{X}$-ray hardness and/or radio flux into three distinct areas (hypersoft, flaring, quiescent). In addition, the flaring state can be further subdivided according to X-ray hardness and the shape of the spectra: flaring/soft X-ray (FSXR), flaring/intermediate (FIM) and flaring/hard X-ray (FHXR). The quiescent state can also be further subdivided into two regions: quiescent and transition. These subdivisions are rather ad hoc since the flaring 
spectra and the quiescent spectra are more or less continuous, but we nevertheless make these distinctions so as to preserve the legacy of previous studies (e.g. Szostek, Zdziarski \& McCollough 2008).

\section{Major flares in Cyg X-3}

In the following we describe the characteristics of a major flaring event in Cyg X-3:

(a) Once Cyg X-3 enters the radio/HXR quenched state, denoted as the hypersoft state in Fig. 1, it will remain in that state for a couple of days up to a month. It is a rare state, comprising $\sim 2-3 \%$ of the overall monitoring live time. Upon emerging from the hypersoft state, Cyg X-3 will always exhibit major flaring (Szostek, Zdziarski \& McCollough 2008; Koljonen et al. 2010).

(b) The HXR and radio switch from an anti-correlation to a correlation when entering the hypersoft state and the HXR flare together with the radio (McCollough et al. 1999). However, major flares differ depending on how strong the accompanying HXR flare is (Koljonen et al. 2010). Variations can also occur within the same flaring episode.

(c) During a major flaring episode, the X-ray spectra evolve from soft to hard (Szostek, Zdziarski \& McCollough 2008; Koljonen et al. 2010). The episode begins in the hypersoft state that is then followed by a rising non-thermal tail in the X-ray spectra (i.e. FSXRFIM states) until the tail exhibits a cutoff (FHXR state). However, the FHXR and FIM states can occur in the opposite order after the peak of the major flare.

(d) The SEDs after a major flare can be fitted with a microquasar model consisting of soft X-ray seed photons from the inner accretion disk, a Comptonizing population of hot electrons and a synchrotron emitting diluted jet. Recently, we have found that the radio emission shows a correlation with the K-band (McCollough et al., in prep.), strengthening the notion of jet emission extending into the IR. Preliminary results from the fitting shows an optically thin radio spectra, strong absorption from IR to SXR and decreasing optical depth as the major flare decay (Koljonen \& McCollough 2008; Koljonen et al., in prep.).

(e) Interestingly, major flares show up as multiple-peaked in the radio (see possible explanation for double-peaked flares in Tammi \& Hovatta 2010).

(f) QPOs are sometimes associated with a decaying major flare; see Fig. 2 where the QPOs detected in van der Klis \& Jansen (1985) are shown in the context of GBI radio monitoring (Koljonen et al., in prep.).

\section{4. $\gamma$-rays in Cyg X-3}

$\mathrm{GeV}$ emission has recently been detected from Cyg X-3 by AGILE (Tavani et al. 2009) and Fermi (Fermi LAT Collaboration et al. 2009; Corbel, these proceedings). The $\gamma$-ray emission arises during specific times characterized by low HXR (Fig. 3) and radio flux and high SXR flux, i.e. the hypersoft state. The $\gamma$-ray flares are observed during the declining phase as well as the rising phase to/from the hypersoft state (Tavani et al. 2009). It is still under debate whether the $\gamma$-rays arise from leptonic (Dubus, Cerutti \& Henri 2010) or hadronic processes (e.g. Romero et al. 2003).

\section{Summary}

Cyg X-3 exhibits complicated spectral behavior and it is only through multi-wavelength observations that more comprehensive insight is gained from this system. Here we have shown that by taking into an account simultaneous radio, soft X-ray, hard X-ray and 


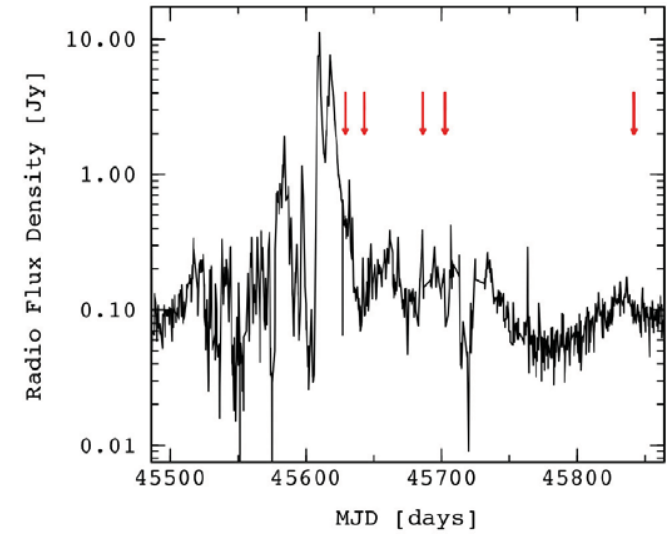

Figure 2. GBI $2.25 \mathrm{GHz}$ lightcurve with red arrows indicating when EXOSAT/ME (van der Klis \& Jansen 1985) observations exhibited QPOs (Koljonen et al., in prep.).

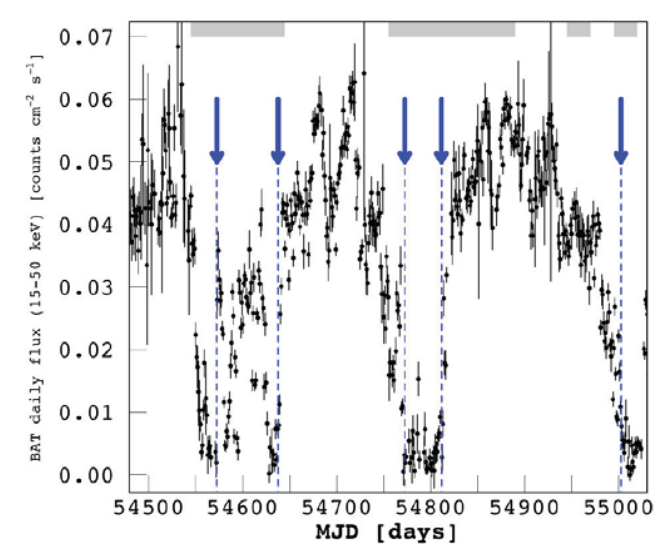

Figure 3. The BAT lightcurve with AGILE coverage (shaded gray bars) and the GeV detections (blue arrows).

$\gamma$-ray observations we can form an unified picture of the overall spectral evolution of the system. The most interesting spectral state probed by this approach is the hypersoft state, a prelude to major flare event in the system, characterized by quenched emission in the radio and hard X-rays, and peak emission in the soft X-rays and $\gamma$-rays. The study of the theoretical implications of these observations is ongoing and a large multi-wavelength collaboration is at the ready to catch this hypersoft state and the accompanying major flaring event over the whole electromagnetic spectrum.

\section{References}

Carpano, S., Pollock, A. M. T., Prestwich, A., Crowther, P., Wilms, J., Yungelson, L., \& Ehle, M. 2007, $A \& \circlearrowleft A, 466, \mathrm{~L} 17$

Dubus, G., Cerutti, B., \& Henri, G. 2010, MNRAS, 404, L55

Fermi LAT Collaboration et al. 2009, Science, 326, 1512

Giacconi, R., Gorenstein, P., Gursky, H., \& Waters, J. R. 1967, ApJ, 148, L119

Koljonen, K. I. I., Hannikainen, D. C., McCollough, M. L., Pooley, G. G., \& Trushkin, S. A. 2010, MNRAS, 406, 307

Koljonen, K. I. I. \& McCollough, M. L. 2008, in proceedings of 7th INTEGRAL Workshop, PoS (Integral08) 075

Mason, K. O., Cordova, F. A., \& White, N. E. 1986, ApJ, 309, 700

McCollough, M. L., Robinson, C. R., Zhang, S. N. et al. 1999, ApJ, 517, 951

Mioduszewski, A. J., Rupen, M. P., Hjellming, R. M. et al. 2001, ApJ, 553, 766

Parsignault, D. R., Gursky, H., Kellogg, E. M. et al. 1972, Nature, 239, 123

Prestwich, A. H., et al. 2007, ApJL, 669, L21

Romero, G. E., Torres, D. F., Kaufman Bernadó, M. M., \& Mirabel, I. F. 2003, A\& A, 410, L1

Szostek, A., Zdziarski, A. A. \& McCollough, M. 2008, MNRAS, 388, 100

Tammi, J. \& Hovatta, T. 2010, International Journal of Modern Physics D, 19, 971

Tavani, M., Bulgarelli, A., Piano, G. et al. 2009, Nature, 462, 620

van der Klis, M. \& Jansen F. A. 1985, Nature, 313, 768

van Kerkwijk, M. H., Charles, P. A., Geballe, T. R. et al. 1992, Nature, 355, 703

Waltman, E. B., Ghigo, F. D., Johnston, K. J., Foster, R. S., Fiedler, R. L., \& Spencer, J. H. 1995, AJ, 110, 290 


\section{Discussion}

KALEMCI: For all the other transients, the flares happen during the hard to soft state transition. Why does it happen the other way around for Cyg X-3?

KolJonen: This is true and Cyg X-3 indeed is a rather bizarre object. At this point, I would speculate the physical scenario behind the observations, but this might be due to the disappearence of the inner disk into the jet during the jet ejection.

Rodriguez: Mirabel argued that HMXRBs would be stationary with respect to the surrounding medium, How much is known of the motions of Cyg X-3?

KolJonen: I don't know about the proper motion Cyg X-3, but maybe James MillerJones can say something more about it. 